Courrier du Centre international Blaise Pascal

16 | 1994

Varia

\title{
Pascal et le cœur
}

\section{Antony McKenna}

\section{OpenEdition}

\section{Journals}

Édition électronique

URL : http://journals.openedition.org/ccibp/588

DOI : $10.4000 /$ ccibp.588

ISSN : 2493-7460

\section{Éditeur}

Centre international Blaise Pascal

\section{Édition imprimée}

Date de publication : 5 avril 1994

Pagination : 3-8

ISSN : 0249-6674

\section{Référence électronique}

Antony McKenna, « Pascal et le cœur », Courrier du Centre international Blaise Pascal [En ligne],

16 | 1994, mis en ligne le 07 janvier 2016, consulté le 19 avril 2019. URL : http://

journals.openedition.org/ccibp/588 ; DOI : 10.4000/ccibp.588

Ce document a été généré automatiquement le 19 avril 2019.

Centre international Blaise Pascal 


\title{
Pascal et le cœur
}

\author{
Antony McKenna
}

1 Un des problèmes les plus complexes dans l'interprétation des Pensées est la définition du «sentiment» et du « cœur »: certes on peut être sensible aux résonances bibliques et augustiniennes de ces termes tels qu'ils sont employés par Pascal ${ }^{1}$, mais des difficultés considérables subsistent, comme l'a souligné récemment Henri Gouhier ${ }^{2}$. En particulier, on comprend mal comment le cœur peut être le siège de sentiments tels que l'amour et la foi, et aussi l'instrument par lequel l'homme appréhende les idées primitives (ou principes) telles que le temps, la dimension, le nombre. Le sentiment, chez Pascal, semble être à la fois émotion et perception. J'envisagerai ici d'abord l'exemple de l'amour, exemple privilégié de « sentiment du cœur » chez Pascal. Ensuite, j'essayerai de comparer l'amour avec d'autres sentiments dans le mécanisme de la psychologie pascalienne, et je tirerai quelques conclusions générales concernant le statut primordial du cœur dans Pensées.

2 Abordons l'amour sous la forme de l'amour-propre. On sait que l'analyse pascalienne de l'amour-propre se fonde sur la doctrine augustinienne des deux amours ${ }^{3}$. Cependant, Pascal apporte une analyse proprement philosophique de ce qui constitue la dépravation du « moi haïssable ». Ce « moi haïssable » se fait « centre de tout » (L. 597 : S. 494) : en cela il déraisonnable et injuste :

...Si nous naissions raisonnables et indifférents, et connaissant nous et les autres nous ne donnerions point cette inclination à notre volonté (L. 420 / S. 680)

En effet, l'amour-propre se heurte à ce que Pascal appelle ici « l'ordre » :

Car tout tend à soi : cela est contre tout ordre. Il faut tendre au général... (L. 420 /

S. 680).

4 Il existe donc un « ordre » raisonnable et juste de l'amour. Quel est-il ? Pascal donne à la doctrine des deux amours une expression laïque :

...Je dis que le cœur aime l'être universel naturellement et soi-même naturellement, selon qu'il s'y adonne, et il se durcit contre l'un ou l'autre à son choix... (L. 423 / S. 680).

5 Ici Pascal oppose l'être individuel du moi à l'être universel : l'être universel est le tout dont l'être individuel fait partie : le «choix» de la volonté injuste est donc de s'aimer 
soi-même au mépris de l'amour qu'elle doit - selon l'«ordre » - à l'être universel. Ce choix n'est pas raisonnable :

Vous avez rejeté l'un [l'amour de l'être universel] et conservé l'autre [l'amour de soi-même] est-ce par raison que vous vous aimez? (L. 423 / S. 680).

6 L'« ordre » consiste donc à aimer le tout dont on fait partie, et cet ordre règne dans tous les domaines de l'activité humaine. Le « désordre »- qui à l'origine est une dépravation de la volonté - consiste à préférer la partie au tout et à concevoir les intérêts de cette partie comme contraires à ceux du tout. Le désordre est une déraison, symptôme d'une dépravation de la volonté.

7 Le rapport du moi individuel à l'être universel fait l'objet aussi de ce "ressouvenir " cartésien :

Je sens que je puis n'avoir point été, car le moi consiste dans ma pensée ; donc moi qui pense n'aurois point été, si ma mère eût été tuée avant que j'eusse été animé, donc je ne suis point un être nécessaire. Je ne suis pas aussi éternel, ni infini, mais je vois bien qu'il y a dans la nature un être nécessaire, éternel et infini (L. 135 / S. 167).

Dans ce fragment l'être du moi est opposé à l'être universel qui est un «être nécessaire, éternel et infini » : c'est-à-dire mon être intelligent est opposé à l'Être intelligent pris sans limitation. Or, on sait que par cet Être intelligent pris sans limitation - Être nécessaire, éternel et infini - Descartes entendait l'Être de Dieu. Pascal reprend cette terminologie ailleurs :

La vraie et unique vertu est donc de se haïr, car on est haïssable par sa concupiscence, et de chercher un être véritablement aimable pour l'aimer. Mais comme nous ne pouvons aimer que ce qui est hors de nous, il faut aimer un être qui soit en nous et qui ne soit pas nous. Et cela est vrai d'un chacun de tous les hommes. Or, il n'y a que l'être universel qui soit tel. Le royaume de Dieu est en nous. Le bien universel est en nous, est nous-mêmes et n'est pas nous. (L. 564 / S. 471).

Dans ces fragments, le rapport du moi individuel à l'Être universel n'est pas seulement un rapport intellectuel fondé sur l'ordre cartésien des raisons, mais un rapport fondée sur l'« ordre » raisonnable (et cartésien) de l'amour.

11 En effet, la définition de l'ordre de l'amour - l'analyse du moi haïssable et de l'Être aimable de Dieu - est fondée très précisément sur la définition cartésienne des passions de l'amour et de la haine :

L'Amour est une émotion de l'âme, causée par le mouvement des esprits, qui l'incite à se joindre de volonté aux objets qui paraissent lui être convenables. Et la Haine est une émotion, causée par les esprits, qui incite l'âme à vouloir être séparée des objets qui se présentent à elle comme nuisibles (Passions, art.79).

Descartes précise alors «ce que c'est que se joindre ou séparer de volonté »: il entend parler du

consentement par lequel on se considère dès à présent comme joint avec ce qu'on aime : en sorte qu'on imagine un tout, duquel on pense être seulement une partie, et que la chose aimée en est une autre (art.80).

13 Cette définition fonde l'ordre pascalien de l'amour : l'ordre qui porte le moi à aimer l'Être universel est conforme à la définition cartésienne de l'amour qui porte la volonté à se joindre au tout dont elle fait partie. De même, le moi «haïssable» est celui qui ne se considère pas comme la partie d'un tout, mais comme un tout séparé :

...en la Haine on se considère seul comme un tout, entièrement séparé de la chose pour laquelle on a de l'aversion (art. 80). 
14 juste - qui permet de dénoncer le désordre du moi haïssable comme une déraison symptomatique de la corruption, comme une «chose monstrueuse,... un enchantement incompréhensible et un assoupissement surnaturel, qui marque une force toute-puissante qui le cause » (L. 427 / S. 681 ) - et cette conception de l'« ordre » se fonde sur les termes mêmes de la définition cartésienne de l'amour.

Cette même définition cartésienne de l'amour lui permet de reprendre ensuite une métaphore paulinienne : d'abord, Pascal définit le tout : « Pour régler l'amour qu'on doit à soi-même, il faut s'imaginer un corps plein de membres pensants, car nous sommes membres du tout, et voir comment chaque membre devrait s'aimer...» (L. 368 / S. 401). Ensuite, le bonheur du membre individuel est défini en fonction de son appartenance au tout : « Pour faire que les membres soient heureux, il faut qu'ils aient une volonté et qu'ils la conforment au corps » (L. 370 / S. 402). Être uni au corps, c'est donc le moyen pour l'être individuel de rejoindre le tout dont il fait partie - l'être universel - et de trouver son propre bonheur dans la soumission à la volonté du tout : « Être membre et n'avoir de vie, d'être et de mouvement que par l'esprit du corps et pour le corps. Le membre séparé ne voyant plus le corps auquel il appartient n'a plus qu'un être périssant et mourant. Cependant, il croit être un tout, et ne se voyant point de corps dont il dépende, il croit ne dépendre que de soi et veut se faire centre et corps lui-même... » (L. 372 / S. 404). C'est là la perversion de la volonté, de l'amour-propre, du moi qui est condamné à l'illusion et au malheur : «Mais n'ayant point en soi de principe de vie, il ne fait que s'égarer et s'étonne dans l'incertitude de son être, sentant bien qu'il n'est pas corps [c'est-à-dire ici, qu'il n'est un tout en lui-même], et cependant ne voyant point qu'il soit membre d'un corps. Enfin, quand il vient à se connaître, il est comme revenu chez soi et ne s'aime plus que pour le corps. Il plaint ses égarements passés. (L. 372 / S. 404) Être membre du corps de membres pensants - image paulinienne de l'église invisible de la communauté des fidèles implique en ce sens une conversion de la volonté égoïste : le moi haïsable est habité par un amour illusoire de soi-même, se prend pour un tout : «Il ne pourrait pas par sa nature aimer une autre chose sinon pour soi-même et pour se l'asservir, parce que chaque chose s'aime plus que tout. Mais en aimant le corps, il s'aime soi-même, parce qu'il n'a d'être qu'en lui, par lui et pour lui. Qui adhoeret Deo unus spiritus est. (L. 372 / S. 404). La métaphore nous fournit ainsi une règle de l'amour juste, amour qui ne se réalise qu'en la foi par laquelle le moi se fond dans l'être universel. « Le corps aime la main, et la main, si elle avait une volonté, devrait s'aimer de la même sorte que l'âme l'aime. Tout amour qui va au-delà est injuste... On s'aime parce qu'on est membre de Jésus-Christ. On aime Jésus-Christ parce qu'il est le corps dont on est membre. Tout est un. L'un est en l'autre... » (L. 372 / S. 404). Tel est le sens de cette formule très forte qui a fait l'objet d'une attaque utilitariste - et très superficielle - de Voltaire dans l'anti-Pascal : «Il faut n'aimer que Dieu et ne haïr que soi » (L. 373 / S. 405).

On voit que la définition cartésienne de l'amour a servi à Pascal à conduire l'interlocuteur d'une conception laïque de la passion ou du sentiment à l'image biblique de l'Église. La religion chrétienne répond en ce sens à l'attente, à l'aspiration du cœur.

C'est là un premier exemple de sentiment où l'on voit très clairement que la psychologie pascalienne a été élaborée dans le contexte créé par les écrits de Descartes : Discours de la méthode (1637), Méditations et Objections (1641), Passions de l'âme (1649) et aussi Lettres (vol. I : 1657). Voyons maintenant s'il y a un rapport entre la définition du sentiment de l'amour et celle d'autres sentiments évoqués dans les Pensées.

Courrier du Centre international Blaise Pascal, 16 | 1994 
18 L'amour est un sentiment, cela va de soi : et son siège est, non pas l'intelligence, l'esprit, mais le «cœur». Mais la mémoire est aussi un sentiment (L. 646 / S. 531) et elle est « nécessaire pour toutes les opérations de la raison » (L. 651 / S. 536). De plus, c'est sur les « connaissances du cœur et de l'instinct » qu'il faut que la raison «s'appuie »: c'est le cœur qui « sent » les principes « comme qu'il y a espace, temps, mouvement, nombres » (L. 110 / S. 142), et c'est en ce sens que «tout notre raisonnement se réduit à céder au sentiment » (L. 530 / S. 455). Les instruments initiaux, les concepts élémentaires, de tous nos raisonnements nous sont fournis par le cœur.

D’où vient ce sentiment des principes? Voyons comment est créé un sentiment chez Pascal.

20 «Car il ne faut pas se méconnaître. Nous sommes automates autant qu'esprit» - « l'automate entraîne l'esprit sans qu'il y pense » (L. 821 / S. 661). C'est l'automate, la Machine, l'habitude du corps qui crée le sentiment. Et, « une fois que l'esprit a vu où est la vérité ", Pascal a recours à ce mécanisme pour ancrer dans le sentiment une conviction qui risquerait de rester trop fragile, trop abstraite, pour ainsi dire, sans l'appui du sentiment. L'automate - l'habitude du corps - nous permet d'acquérir « une créance plus facile» - «car d'en avoir les preuves toujours présentes, c'est trop d'affaire»: «l'habitude [...] sans violence, sans art, sans argument, nous fait croire les choses et incline toutes nos puissances à cette croyance, en sorte que notre âme y tombe naturellement» (L. 821 / S. 661). Et ce mécanisme nous est familier dans le vocabulaire qui caractérise l'argument du «pari » : c'est « l'abêtissement » : " naturellement même cela vous fera croire et vous abêtira » (L. 418 / S. 680). C'est le mécanisme par lequel les habitudes du corps plient la Machine, matent les passions rebelles et ancrent dans le sentiment du cœur une conviction de l'esprit. "Qui s'accoutume à la foi la croit...» (L. 419 / S. 680). C'est la loi de toutes nos opinions, de toutes nos convictions, de tous nos sentiments : "La coutume est notre nature » (L. 419 / S. 680). Le corps joue donc un rôle primordial dans la naissance des sentiments.

21 Se pose alors un problème de cohérence. Dans le fragment L. 110 / S. 142, nous l'avons vu, le cœur sent les principes et c'est sur les connaissances du cœur et de l'instinct que la raison doit s'appuyer. Quel rapport entre ce sentiment des principes et l'automate qui crée les sentiments dans les fragments que je viens de citer (L. 82l, 418 / S. 661, 680) ? Un premier indice nous est fourni par le début même du « pari » :

L'âme est jetée dans le corps où elle trouve nombre, temps, dimensions. Elle raisonne là-dessus et appelle cela nature, nécessité, et ne peut croire autre chose. (L. 418 / S. 680).

Cette formule confirme le rôle du corps dans la naissance du sentiment - rôle exprimé d'ailleurs sans équivoque dans les fragments cités ci-dessus. Mais qu'est-ce à dire : « l'âme trouve dans le corps »? Le fragment des « Deux Infinis » fournit une clef. Pascal y propose une sorte de cheminement épistémologique par lequel nous aboutissons à la définition des principes :

...toutes les sciences sont infinies en l'étendue de leurs recherches. Car qui doute que la géométrie, par exemple, a une infinité d'infinité de propositions à exposer? Elles sont aussi infinies dans la multitude et l'infinité de leurs principes. Car qui ne voit que ceux qu'on propose pour les derniers ne se soutiennent pas d'eux-mêmes et qu'ils sont appuyés sur d'autres qui, en ayant d'autres pour appui, ne souffrent jamais de dernier? Mais nous faisons des derniers qui paraissent à la raison comme on fait dans les choses matérielles, où nous appelons un point indivisible celui au- 
delà duquel nos sens n'aperçoivent plus rien, quoique divisible infiniment et par sa nature. (L. 199 / S. 230). notre esprit est incapable de saisir ces principes infiniment délicats; les principes que nous fournit le cœur ou le corps correspondent donc à ce que nous appelons « un point indivisible » dans les choses matérielles. En d'autres termes, l'évidence des principes est un effet de la limitation de la vue claire de l'esprit, et cette limitation est imposée par le corps. "Trouver dans le corps" signifie donc ici que ces principes sont ceux qui nous apparaissent comme «premiers» (ou "derniers») parce que le corps obscurcit la vue claire de l'esprit. En ce sens, les principes sont ceux qui apparaissent à un esprit uni à un corps. Le corps détermine ainsi notre vision des choses: nous analysons le monde selon nos "principes », nous appelons cela "nature », «nécessité », mais cette " nécessité » n'est que celle de notre nature et non pas du monde que nous percevons. Ce n'est que parce qu'elle se trouve unie à un corps que l'âme pense en termes de nombres, temps, dimensions. Ce sont des concepts issus de l'union de l'âme et du corps : le sentiment est donc le mode de connaissance qui caractérise un être constitué d'une âme et d'un corps. Ce fragment confirme à mes yeux le lien de dépendance qui lie les sentiments au corps et confirme du même coup que nous n'avons pas affaire à un "sentiment d'évidence ", à une « intuition intellectuelle » : jamais, chez Pascal, l'esprit ne fonctionne indépendamment du corps. L'âme n'a pas, comme chez Descartes, ses « espèces à part ». Au contraire, les idées primitives sont "trouvées" dans le corps et "senties» par le cœur. Cette perspective me semble confirmer la dimension anti-cartésienne du sentiment pascalien.

Le désordre de l'amour est une déraison, disions-nous. De même, la saisie des principes «trouvés » dans le corps est une déraison imposée par notre nature actuelle, dominée par le corps, "pareille à celle des animaux» (L. 117 / S. 149). Le sentiment pascalien de l'amour est conforme à la définition de la passion cartésienne. De même, sur le plan de la connaissance, le sentiment pascalien est conforme à la définition cartésienne des «sentiments de douleur, de soif, de faim, etc.» qui sont «certaines façons confuses de penser qui proviennent et dépendent de l'union et comme du mélange de l'esprit et du corps » (AT. IX, 64 ; Alquié, II, 492), - sentiments qui permettent à Descartes de conclure précisément « que je ne suis pas seulement logé dans mon corps, ainsi qu'un pilote en son navire, mais, outre cela, que je lui suis conjoint très étroitement et tellement confondu et mêlé, que je compose un seul tout avec lui. » (ibid.).

Or, l'évidence et la certitude des intuitions intellectuelles dépendent, dans la philosophie cartésienne, de la liberté de la volonté, qui peut toujours suspendre le jugement et ne pas embrasser les idées obscures ou confuses. De même, dans le traité des Passions de l'âme, cette liberté de la volonté par rapport aux perceptions confuses fonde notre capacité de contrôler les passions, et fonde donc aussi la morale de la générosité. Les perceptions des passions sont obscures et confuses : devant une telle obscurité et une telle confusion, le libre arbitre peut toujours et doit toujours suspendre le jugement, car il est toujours et absolument en notre pouvoir. Or, de l'analyse pascalienne du rôle du sentiment, il s'ensuit que nous n'avons que des idées obscures ou confuses, en ce sens qu'elles ne sont pas engendrées par une pure intelligence, mais par l'union de l'âme et du corps : elles sont toutes fondées sur le sentiment. Devant de telles perceptions obscures et confuses, Descartes s'impose la suspension du jugement. Dans la perspective pascalienne, une telle suspension est impossible : 
L'esprit croit naturellement et la volonté aime naturellement de sorte qu'à faute de vrais objets il faut qu'ils s'attachent aux faux (L. 661 / S. 544).

La psychologie cartésienne est décapitée : dans le domaine de la connaissance, comme dans celui de l'amour, l'homme est livré à ses sentiments. Or,

la fantaisie est semblable et contraire au sentiment, de sorte qu'on ne peut distinguer entre ces contraires... Il faudrait avoir une règle. La raison s'offre, mais elle est ployable à tous sens. Et ainsi il n'y en a point. (L. 530 / S. 455).

Le lien entre le sentiment et le corps est ainsi confirmé, car la fantaisie est fruit de l'imagination, de la faculté qui transforme les données des sens en images pour les livrer au travail de l'esprit. C'est, en effet, l'imagination qui a créé une «seconde nature » en l'homme, et c'est dans cette "nature» dominée par la coutume, par le corps, que le sentiment joue un rôle primordial, non seulement dans le domaine des affections qui est le sien, mais aussi dans celui des connaissances.

Dès lors le sentiment est dépourvu de garantie métaphysique. Tandis que Descartes fondait sur la véracité divine la garantie que l'évidence ne pouvait le tromper (car Dieu est auteur de ma nature et c'est Lui qui me tromperait s'il m'avait créé de telle sorte que je sois obligé par ma nature d'acquiescer, sous la force de l'évidence, à des choses qui s'avéreraient fausses), l'épistémologie pascalienne est dépourvue de cette garantie: «Dieu tente mais il n'induit pas en erreur » (L. 850 / S. 431), c'est-à-dire Dieu ne nous oblige pas à prendre nos certitudes pour des vérités absolues qui s'imposeraient à Dieumême; l'homme peut reconnaître ainsi son incapacité à atteindre par ses propres forces la vérité, sans que Dieu le trompe, sans que Sa véracité soit mise en cause. L'homme reconnaît sa misère : il reconnaît qu'en perdant sa première nature il a perdu sa capacité initiale de se détacher de l'emprise du corps et de l'erreur.

L'homme est livré au sentiment. Quelle que soit son intensité, sa certitude intérieure (voir le Mémorial : «Certitude, certitude, sentiment, joie, paix » (L. 913 / S. 742), le sentiment ne peut qu'être senti ; il ne peut pas être démontré. Dieu reste un Dieu caché.

Mon commentaire - ce n'est pas une surprise - confirme le statut primordial du cœur dans la psychologie pascalienne, mais il permet aussi de saisir le lien entre les domaines de l'affectivité et de la connaissance. Dans les deux cas, le sentiment est conçu par Pascal dans le contexte de la psychologie cartésienne. Le sentiment se substitue à l'intuition intellectuelle de Descartes, et, dans la mesure où le sentiment dépend de notre nature corporelle, il constitue une objection radicale au rationalisme cartésien.

31 D'autre part, Pascal appuie sa conception du sentiment sur la définition cartésienne de ces sentiments qui sont "certaines façons confuses de penser qui proviennent et dépendent de l'union et comme du mélange de l'esprit avec le corps ». Il rend ainsi à l'imagination le rôle central qui est le sien dans la psychologie gassendiste : nous sommes incapables de distinguer entre le sentiment vrai et la fantaisie fausse. Nos sentiments, liés à notre corps, sont dépourvus de garantie et ne valent que pour nous.

Enfin, le vocabulaire cartésien de la passion permet à Pascal de rejoindre les grands thèmes de la doctrine augustinienne. Il fournit ainsi le passage entre la psychologie laïque du cartésianisme (ou du gassendisme) et la doctrine augustinienne de la perversion de la nature et la théologie augustinienne des deux amours. De même que le statut du coeur défini dans la perspective d'une psychologie cartésienne décapitée lui permet de reprendre les connotations augustiniennes et bibliques de ce terme ( Dieu sensible au cœur »), de même la définition cartésienne de la passion de l'amour lui permet de 
rejoindre le langage paulinien et d'exprimer dans la perspective d'un ordre juste et raisonnable la perversité de l'amour-propre et le bonheur de l'amour juste - de l'amour qui se réalise dans le corps mystique du Christ. Le cœur réalise ainsi le sens de la Figure, car le corps n'est que figure. Ainsi se confirme le règne du corps qui est punition et pénitence et qui figure la voie du salut.

\section{NOTES}

1. Voir Ph. Sellier, Pascal et Saint Augustin, Paris, p. 117-139

2. H. Gouhier, Blaise Pascal, conversion et apologétique, Paris, 1986, chap. 3.

3. Ph. Sellier, op. cit., p. 140-151.

INDEX

Mots-clés : Pascal, Pensées, cœur, sentiment, amour

Keywords : Pascal, Pensées, heart, feeling, love

\section{AUTEUR}

\section{ANTONY MCKENNA}

Professeur de littérature française

Université de Saint-Etienne, Institut d'histoire de la pensée classique (CNRS UMR 5037) 